\title{
Expression and localisation of FSHR, GHR and LHR in different tissues and reproductive organs of female yaks
}

\author{
H. Chen, Y. Cui, S. Yu \\ College of Veterinary Medicine, Gansu Agricultural University, Gansu, China \\ [Received: 31 August 2017; Accepted: 11 September 2017]
}

Background: This study aimed to investigate the expression and localisation of follicle stimulating hormone receptor/growth hormone receptor/luteinising hormone receptor (FSHR/GHR/LHR) in different tissues and examine the regulatory effects of FSHR/GHR/LHR in the reproductive organs of female yaks during luteal phase. Materials and methods: The quantitative real-time polymerase chain reaction (qRT-PCR) and immunohistochemistry assays were utilised to analyse the expression and localisation of FSHR/GHR/LHR in different tissues on female yaks.

Results: The GRT-PCR results showed that the MRNA expressions of FSHR/GHR/ ILHR were significantly different in the non-reproductive organs $(p<0.01)$; the highest expression level was observed in the kidney, cerebellum and lung, whereas the lower expression level was observed in the liver and spleen. Immunohistochemistry assay results showed that FSHR/GHR/LHR were located in kidney tubules, Purkinje cells, cerebellar medulla, alveolar cells and hepatocytes. In addition, the expression levels of FSHR and GHR were considerably higher than LHR in the reproductive organs of female yaks during luteal phase $(p<0.01)$. FSHR/GHR/LHR were located in cardiac muscle cells, cerebellar medulla, and theca cell lining of reproductive organs. Furthermore, the expression level of FSHR was higher than those of GHR and LHR in all examined tissues.

Conclusions: Therefore, the expression and localisation of FSHR/GHR/LHR possibly helped to evaluate the effects of them in tissue specific expression on female yaks, investigate the function and mechanism of FSHR/GHR/LHR in the reproductive organs of female yaks during luteal phase. (Folia Morphol 2018; 77, 2: 301-309)

Key words: expression and localisation, female yaks, FSHR/GHR/LHR, tissue

\section{INTRODUCTION}

Yak is the ancient uniquely species of terrestrial cattle, which few people have known up to now [25]. Follicle stimulating hormone receptor (FSHR) and growth hormone receptor (GHR) are proteins synthesised and secreted by the pituitary gland, which play important roles in the maturation of follicles. Recent study showed that the AR/miR-126* axis exerts synergetic effects to regulate FSHR expression and apoptosis in PGCs [4]. Studies indicated that profiling of FSHR negative allosteric modulators on LH/CGR reveals biased antagonism with implications in steroidogenesis [2]. In addition, genetic markers might be composed of FSHR, bone morphogenetic protein receptors (BMPR-IB) and bone morphogenetic protein (BMP-15) in multi-gene pyramiding for enhance lit-

Address for correspondence: Dr. S. Yu, College of Veterinary Medicine, Gansu Agricultural University, Lanzhou 730070, Gansu, China, e-mail: sjyu@163.com 
ter size in sheep husbandry [22]. The expressions of FSHR and GHR were significantly different in different animals, and the report found that the decreased expression of GHR might hinder the expression of EGFR and EGFR-AS1 in vivo and in vitro. However, the high expression of EGFR promotes the increase in GHR in hepatocellular carcinoma [18]. The combination of postbiotics and inulin is beneficial to the expression of the GHR mRNA in broiler chickens, and GHR may be a candidate marker to improve meat production of Japanese Black cattle. Holstein/Japanese Black crosses carried the GHR short allele from their sire $[11,16]$.

Luteinising hormone receptor (LHR) is an important reproductive hormone receptor in mammals. In recent years, more and more studies reported that LHR existed in extragonadal tissues such as gastric smooth muscle cell, cell wall, submandibular gland and some malignant tumour cells surface [17]. The expression of LHR is not only found in the ovary, but also in other organs, such as uterus and oviduct of mice, human, cattle, pig, and so on [13, 28]. The study by Marson et al. [15] showed that the obvious effects of polymorphisms of LHR and FSHR genes on sexual precocity in a Bos taurus $\times$ Bos indicus beef composite population.

FSHR/GHR/LHR play key regulatory roles in reproductive organs. There was no difference in the expression of GHR between the breed and the ovulatory status. In addition, the expression of GHR was not correlated with the change of insulin-like growth factor (IGF-I) concentration postpartum in the liver [20]. FSHR has significance regarding the modulation of animal reproductive functions $[10,23]$. Moreover, previous studies found that the FSH could accelerate the maturation rate of sheep oocytes and reduce their apoptosis rate, increase the expression levels of FSHR, LHR and gonadotropin releasing hormone receptor (GnRHR) mRNAs, and strengthen expressions of FSHR and GnRHR proteins [21].

There are still no authoritative reports about the expression of FSHR/GHR/LHR in different tissues of female yaks. In the present study, based on the methods of tissue isolation, expression and localisation in various tissues and organs, we investigated the effects of FSHR/GHR/LHR in the female reproductive organs of yaks. Furthermore, we clarified the relationship between the reproductive function and different tissues, and explored the differential expression levels of FSHR/GHR/LHR in different tissues and reproductive organs by quantitative real-time polymerase chain reaction (qRT-PCR) and immunohistochemical methods. These results could provide the guidance of understanding the function and mechanism of FSHR/ /GHR/LHR in the reproductive organs of female yaks during luteal phase, as well as might lay a foundation for the further study of the physiological functions of different tissues in female yaks.

\section{MATERIALS AND METHODS}

Animals and tissue samples

The female yaks were sampled from the Tibetan plateau in a Qinghai region pasture, Qinghai, China. A total of 6 healthy adult female yaks were killed painlessly and samples were collected including different tissues and reproductive organs. These samples were putted into liquid nitrogen after wrapped with foil. Animal welfare and experimental procedures were performed in accordance with the Guide for the Care and Use of Laboratory Animals (Ministry of Science and Technology of China, 2006) and approved by the Animal Ethics Committee of Gansu Agricultural University.

\section{Determination of FSHR/GHR/LHR expression}

Primers design. The primers based on the FSHR/ /GHR/LHR sequences of cattle (NM-174061.1, NM$176608.1, \mathrm{U}-41414.1)$, and $\beta$-actin sequence of yak (DQ838049.1) were designed by Primer Premier 5.0 software. Then the primers were synthesised by Huada gene research centre (Beijing). PCR primers were performed as indicated in Table 1.

RNA reverse transcription. Total ribonucleic acid (RNA) was extracted using the TRIZOL reagent (Invitrogen, Vienna, Austria) and treated with RNase-free DNase I (Promega) to remove possible contaminating DNA. The purity of the total RNA was estimated by the $\mathrm{OD}_{260} / \mathrm{OD}_{280}$ ratio (1.9-2.0). RNA concentrations were determined with the spectrophotometer (GE, USA) using the $O D_{260} \mathrm{~nm}$. Six randomly chosen control samples without transcriptase (RT-negative) were used as control for reverse transcription step.

Gene expression of FSHR/GHR/LHR in different tissues. The qRT-PCRs of LHR and $\beta$-actin were performed with complementary deoxyribonucleic acid (cDNA) using Light Cycler 480. The total volume was $20.0 \mu \mathrm{L}$, which included $1.0 \mu \mathrm{L}$ of cDNA (equivalent to $50 \mathrm{ng}$ reverse-transcribed total RNA), $10.0 \mu \mathrm{L}$ GoTaqGreenMasterMix (TransGen Biotech), $0.5 \mu \mathrm{L}$ of upstream and downstream primers (10 pM), and then Nuclease-FreeWater $(8 \mu \mathrm{L})$. The conditions of 
Table 1. Primer sequences of target and house-keeping genes

\begin{tabular}{lccc}
\hline Primer name & Sequence $\left(\mathbf{5}^{\prime} \rightarrow \mathbf{3}^{\prime}\right)$ & $\mathbf{T m}\left({ }^{\circ} \mathbf{C}\right)$ & Note \\
\hline FSHR-R & ATCACAGCCTCCTCCA & 50.0 & qRT-PCR \\
FSHR-F & AACTCACAGTCCCCCG & 48.5 & qRT-PCR \\
GHR-R & CAGTCTCAACGAGTACATCGGAAC & 56.0 & qRT-PCR \\
GHR-F & GGGAACCACCACCCAATACAG & 56.0 & \\
LHR-R & CACTCCCTGTCTGCCAGTCTAT & 58.0 & qRT-PCR \\
LHR-F & CTTCTCATGTGCAACCTCTCC & 58.0 & \\
GAPDH-R & ACATACTCAGCACCAGCATCA & 60.0 & 60.0 \\
GAPDH-F & TCAACGGCACAGTCAAGG & & \\
\hline
\end{tabular}

qRT-PCR — quantitative real-time polymerase chain reaction; $\mathrm{Tm}$ — temperature

PCR cycling were as follows: denaturation $5 \mathrm{~min}$ at $95^{\circ}$, and then the best condition of cycle were repeated 45 times $\left(95^{\circ}\right.$ for $10 \mathrm{~s}, 60^{\circ}$ for $\left.15 \mathrm{~s}\right)$; finally, elongation $15 \mathrm{~s}$ at $72^{\circ}$. Each sample was repeated 3 times, followed the fluorescence measurement was continuously performed the melting analysis by $65^{\circ}$ for $10 \mathrm{~s}$, and at rate of per second $0.1^{\circ}$ up to $95^{\circ}$ for slowly heat. For each sample, the expression level of LHR was calculated using $2^{-\Delta \Delta C t}$ method.

\section{Immunohistochemical assays}

The main organs and tissues of the female yaks were fixed in $4 \%$ paraformaldehyde solution at room temperature for 7 days. Tissue pieces were clipped and paraffin embedded, then the sections were sliced, dried and saved.

To investigate FSHR/GHR/LHR expression levels by immunohistochemical method, samples were dewaxed using dimethyl benzene and then dehydrated with an ascending alcohol gradient. These sections were rehydrated, and the antigen was repaired with $3 \%$ deionised $\mathrm{H}_{2} \mathrm{O}_{2}(15-20 \mathrm{~min})$ and sealed with rabbit serum (15-20 $\mathrm{min})$. After overnight incubation at $4^{\circ} \mathrm{C}$ with the primary rabbit anti-FSHR, anti-GHR and anti-LHR monoclonal antibodies (1:300, Bioss, Beijing), then those sections were incubated with the secondary antibody. The labelled samples were counterstained with 3-3'-diaminobenzidine [6]. The images were analysed by Image-ProPlus 6.0, using absorbance analysis of integral optical density of FSHR/GHR/LHR positive reaction in different tissues.

\section{Statistical analysis}

Data were analysed by SPSS software with the lysergic acid diethylamide method (Statistical Package for the Social Sciences, version 21.0 for Windows;
SPSS Inc., Chicago, IL, USA). The other data were analysed by one-way ANOVA and Duncan's post hoc test. P-values between groups less than 0.05 were regarded to be statistically significant [7].

\section{RESULTS}

Expression and distribution of FSHR/GHR/LHR in the non-reproductive organs of female yaks

FSHR/GHR/LHR expression in the non-reproductive organs. To investigate the FSHR/GHR/LHR genes expression in the tissue of the female yaks, qRT-PCR was used with the total RNA isolated from yak's different tissues as controls. The FSHR/GHR/LHR genes were expressed at different levels in the non-reproductive organs, including the kidney, cerebellum, lung, liver, and spleen (Fig. 1). First, the highest expression level of FSHR was observed in the kidney, followed by the cerebellum and lung. In contrast, the lowest expression level was in the spleen, followed by the liver. Second, the highest expression level of GHR was observed in the kidney, followed by the cerebellum and lung. In contrast, the lowest expression level was in the liver, followed by the spleen. Finally, the highest expression level of LHR was observed in the kidney, followed by the cerebellum and lung. In contrast, the lowest expression level was in the spleen, followed by the liver.

In addition, the expression levels of FSHR and LHR were considerably higher than GHR in almost all tested tissues $(p<0.01)$, except the liver and spleen; the results are shown in Table 2 . The expression level of LHR was higher than those of FSHR and GHR in the liver, and GHR was higher than those of FSHR and LHR in the spleen.

FSHR/GHR/LHR distribution in the non-reproductive organs. As shown in Figure 2, FSHR/GHR/LHR 


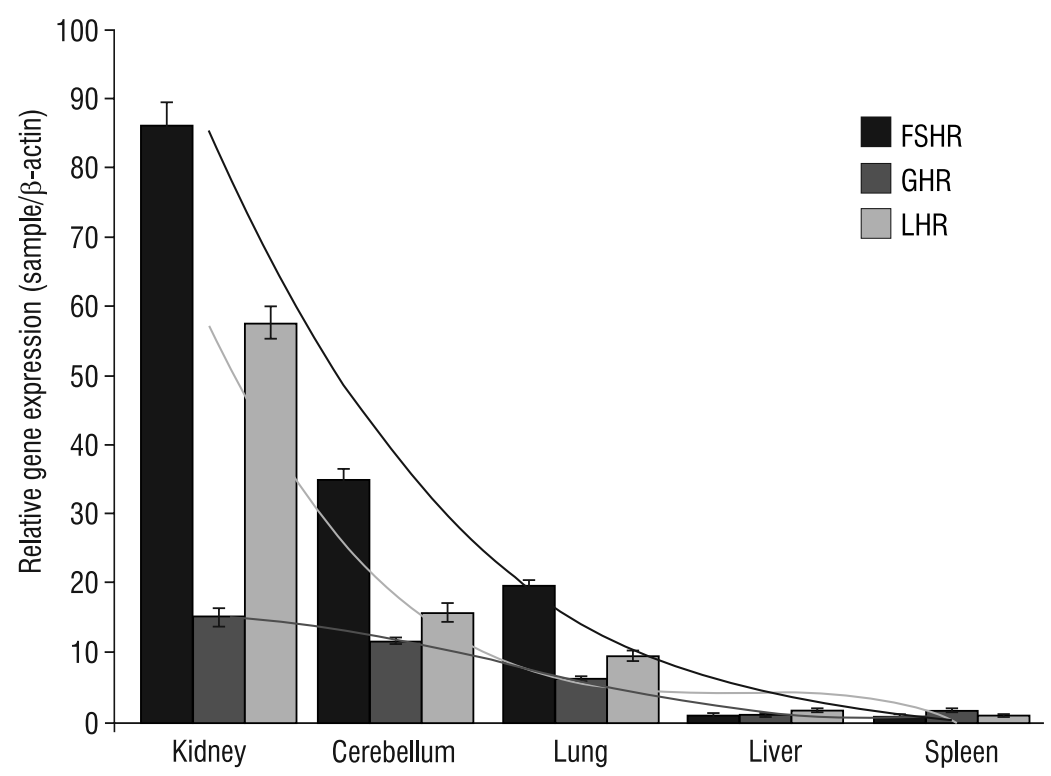

Figure 1. The gene expression levels of follicle stimulating hormone receptor/growth hormone receptor/luteinising hormone receptor (FSHR/GHR/LHR) in non-reproductive system of female yaks. The results of real-time polymerase chain reaction. The different letters mean significant difference between the groups $(p<0.01)$. The same letters mean no difference between the groups $(p>0.05)$.

Table 2. Quantitative real-time polymerase chain reaction (qRT-PCR) results of FSHR/GHR/LHR gene in non-reproductive system of yak

\begin{tabular}{|c|c|c|c|}
\hline Tissues and organs & $\Delta \mathbf{C T}$ & $\Delta \Delta \mathrm{CT}\left(\Delta \mathrm{CT}_{\text {sample }}-\Delta \mathrm{CT}_{\text {ovary/oviduct }}\right)$ & $2^{-\Delta \Delta C T}$ \\
\hline \multicolumn{4}{|l|}{ FSHR } \\
\hline Kidney & $-11.053 \pm 3.3270$ & $-6.4300 \pm 3.3270$ & 86.223 \\
\hline Cerebellum & $-9.7566 \pm 1.4246$ & $-5.1333 \pm 1.4246$ & 35.098 \\
\hline Lung & $-8.9233 \pm 0.8292$ & $-4.3000 \pm 0.8292$ & 19.698 \\
\hline Liver & $-4.6233 \pm 0.3096$ & $0 \pm 0.3096$ & 1 \\
\hline Spleen & $-4.5088 \pm 0.2236$ & $-0.1145 \pm 0.2236$ & 1.0823 \\
\hline \multicolumn{4}{|l|}{ GHR } \\
\hline Kidney & $-8.5480 \pm 1.4024$ & $-3.9247 \pm 1.4024$ & 15.246 \\
\hline Cerebellum & $-8.1785 \pm 0.3310$ & $-3.5552 \pm 0.3310$ & 11.755 \\
\hline Lung & $-7.2824 \pm 0.3012$ & $-2.6591 \pm 0.3012$ & 6.3207 \\
\hline Liver & $-4.7912 \pm 0.2431$ & $-0.1679 \pm 0.2431$ & 1.2339 \\
\hline Spleen & $-5.2988 \pm 0.1982$ & $-0.6765 \pm 0.1982$ & 1.5982 \\
\hline \multicolumn{4}{|l|}{ LHR } \\
\hline Kidney & $-10.475 \pm 2.3387$ & $-5.8517 \pm 2.3387$ & 57.747 \\
\hline Cerebellum & $-8.6136 \pm 1.2136$ & $-3.9903 \pm 1.2136$ & 15.893 \\
\hline Lung & $-7.8834 \pm 0.6222$ & $-3.2601 \pm 0.6222$ & 9.5800 \\
\hline Liver & $-5.3034 \pm 0.2206$ & $-0.6801 \pm 0.2206$ & 1.6021 \\
\hline Spleen & $-4.7478 \pm 0.1785$ & $-0.1245 \pm 0.1785$ & 1.0900 \\
\hline
\end{tabular}

FSHR — follicle stimulating hormone receptor; GHR — growth hormone receptor; LHR — luteinising hormone receptor

were mainly detected in almost all of the non-reproductive organs. FSHR was detected in tubules of kidney, Purkinje cells and medulla of cerebellar, red pulp of spleen, alveolar cell and hepatocytes. GHR was observed in distal and proximal convoluted tubule of kidney, cortex and medulla of cerebellar. LHR was 


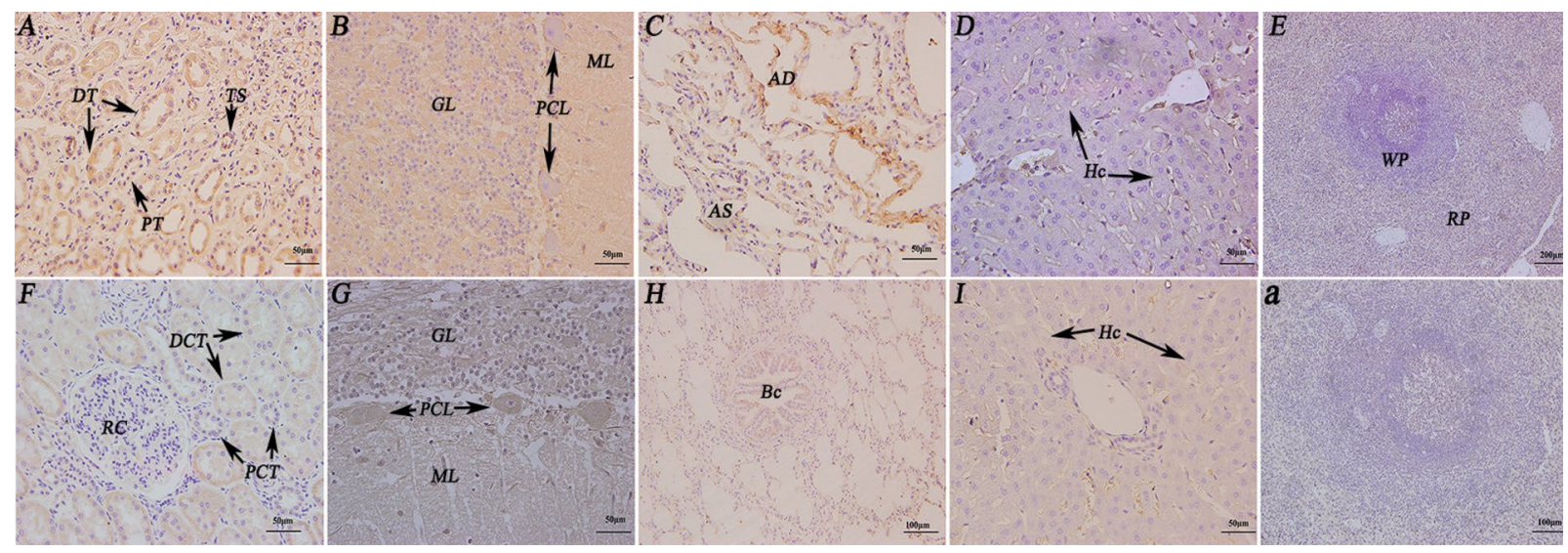

Figure 2. Immunohistochemistry localisation of follicle stimulating hormone receptor/growth hormone receptor/luteinising hormone receptor (FSHR/GHR/LHR) in the non-reproductive system of female yaks. A. Positive staining for FSHR was observed in the tubules of the kidney; B. Positive staining for FSHR was observed in cerebellar medulla and Purkinje cells; C. Positive staining for FSHR was observed in the alveolar cell of the lung; D. Positive staining for FSHR was observed in hepatocytes of the Bos grunniens liver; E. Positive staining for FSHR was observed in the red pulp of spleen; F. Positive staining for GHR was observed in the tubules of the kidney; G. Positive staining for GHR was observed in cerebellar medulla and Purkinje cells; H. Positive staining for LHR was observed in the bronchi of the lung; I. Positive staining for LHR was observed in hepatocytes of the Bos grunniens liver; $\mathbf{a}$. The control sections collected from the spleen of yak, without immunoreactions (negative control); TB — terminal bronchiole; Hc — hepatocyte; CV — central vein; CMF — cardiac muscle fibres; DCT — distal convoluted tubule; $\mathrm{PCL}$ — Purkinje cell layer; $\mathrm{RG}$ — renal glomerulus; $\mathrm{Bf}$ — biofilm; Tc — trabecula; $\mathrm{RP}$ — red pulp; WP — white pulp; $\mathrm{CM}$ — cerebellar medulla; $\mathrm{ML}$ — molecular layer; $\mathrm{GL}$ — granular layer; $\mathrm{PCT}$ — proximal convoluted tubule.

not only observed in alveolar cell and bronchiole of lung, but also in hepatocytes of the liver. Therefore, the FSHR/GHR/LHR were mainly concentrated on the cell membrane and in the cytoplasm, not in the nucleus. In addition, spleen tissue was used as a positive control in immunohistochemical examination. Nevertheless, the slight negative immunostaining of FSHR/GHR/LHR was observed in the liver and spleen. The immunostaining was not observed in glomerulus and white pulp of the kidney and spleen (Fig. 3).

\section{Expression and distribution of FSHR/GHR/LHR in the reproductive organs of female yaks during luteal phase}

FSHR/GHR/LHR expression in the reproductive organs during luteal phase. The results are shown in Figure 4. First, the highest expression level of FSHR was observed in the uterus, followed by the oviduct. In contrast, the lowest expression level was in the ovary. Second, the highest expression level of GHR was observed in the oviduct, followed by the uterus. In contrast, the lowest expression level was in the ovary. However, the expression level of LHR was not observed significant in the uterus, ovaries and oviducts of the yaks (Table 3). FSHR/GHR/LHR expression levels showed significant differences in the female yaks' reproductive organs $(p<0.01)$.
FSHR/GHR/LHR distribution in the reproductive organs during luteal phase. FSHR/GHR/LHR were mainly detected in almost all of the reproductive organs during luteal phase. FSHR and GHR were detected in epithelial cell, lamina propria and circular muscle layer of uterus, mucous plica of oviduct, stratum granulosum of ovary. However, LHR was not only observed in epithelial cell and muscular layer of uterus, mucous plica of oviduct, but also in stratum granulosum, theca externa of follicle of the ovary. Thereby, the FSHR/GHR/LHR were mainly concentrated on the cell membrane, not in the nucleus. In addition, immunohistochemical examination of ovary tissue was used as a positive control.

In conclusion, LHR positive reaction was weaker than FEHR and GHR in reproductive organs of female yaks during luteal phase. Furthermore, the immunostaining was not observed in vertical muscle layer and theca externa of follicle of the uterus and the ovary.

\section{DISCUSSION}

Few studies focused on the expression and localisation of FSHR/GHR/LHR in different tissues of female yaks during luteal phase. Integrated optical density analysis showed that FSHR and LHR were expressed in the pineal gland, hypothalamus, pituitary gland, and gonads of female yaks. FSHR was mainly expressed in the pituitary gland and ovaries, whereas LHR was 


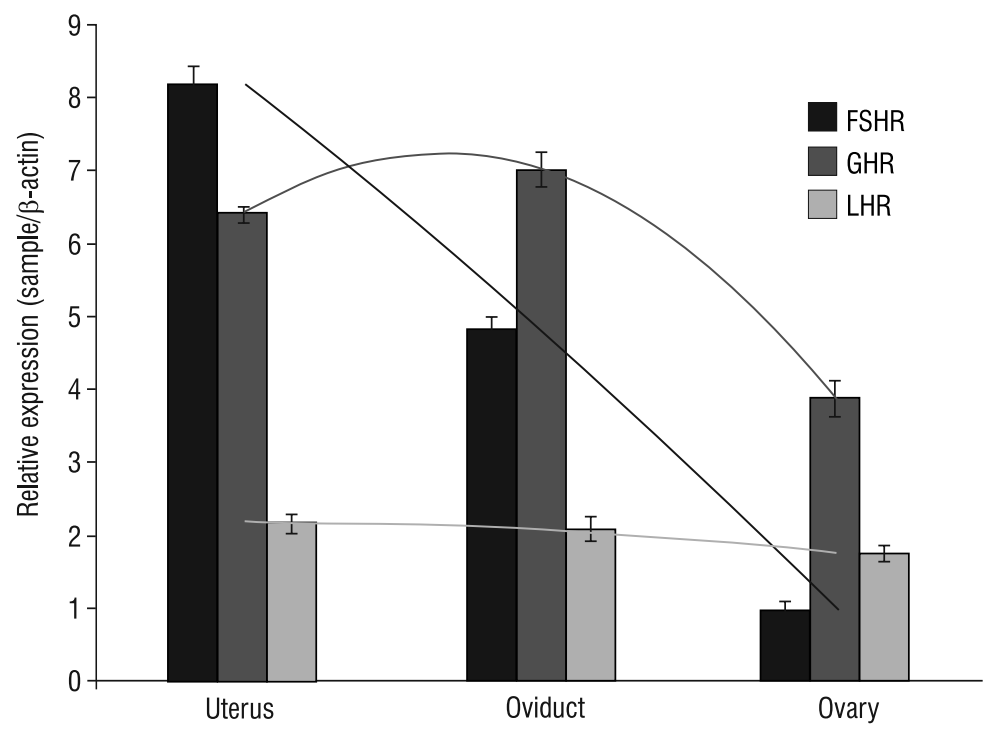

Figure 3. The gene expression levels of follicle stimulating hormone receptor/growth hormone receptor/luteinising hormone receptor (FSHR/GHR/LHR) in reproductive system of yak under follicular phase. The results of real-time polymerase chain reaction. The different letters mean significant difference between the groups $(p<0.01)$. The same letters mean no difference between the groups $(p>0.05)$.
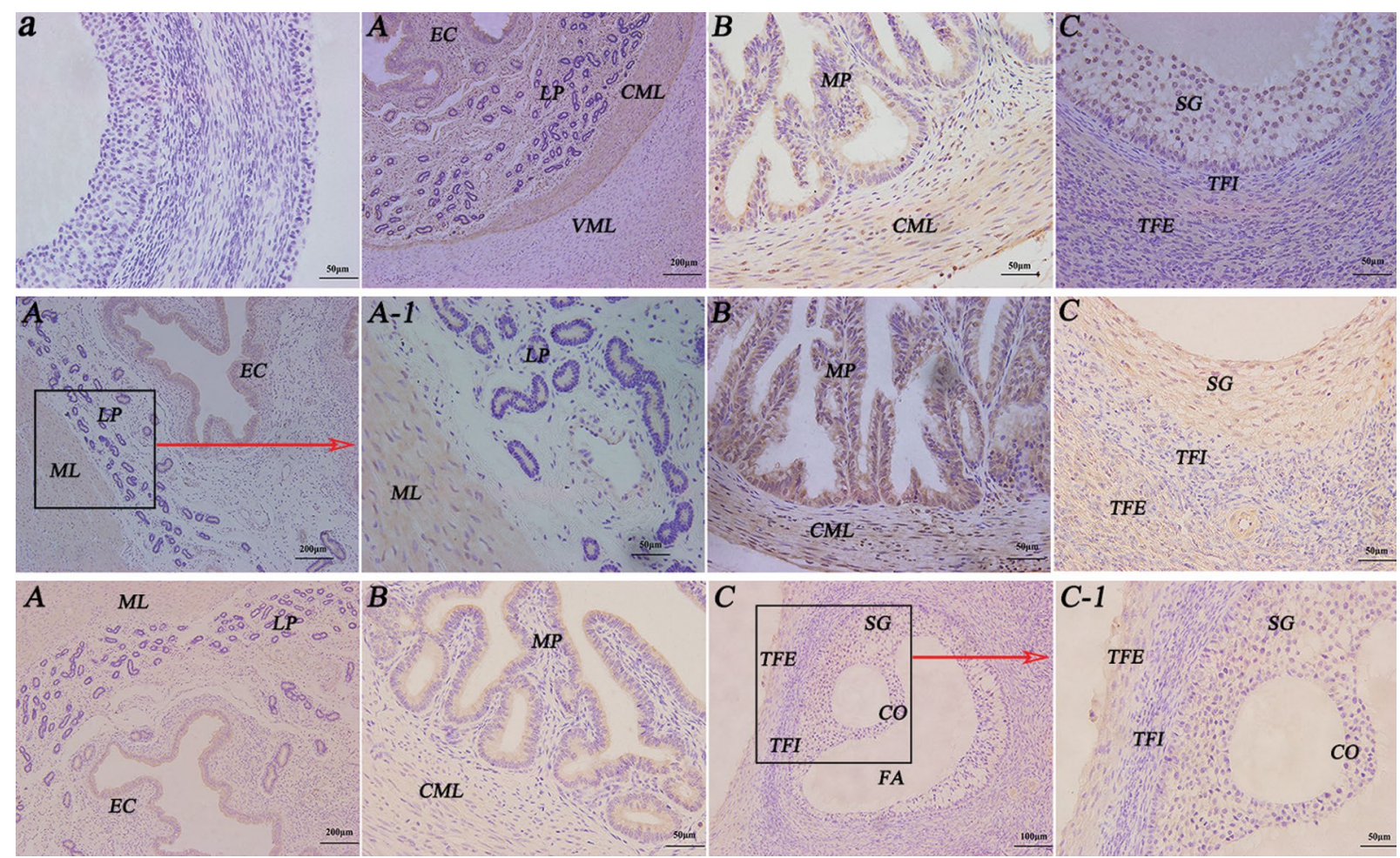

Figure 4. Immunohistochemistry localisation of follicle stimulating hormone receptor/growth hormone receptor/luteinising hormone receptor (FSHR/GHR/LHR) in the reproductive system of female yaks. First-line: a. The control sections collected from the uterus of female yak, without immunoreactions (negative control); A. Positive staining for FSHR was observed in epithelial cell, lamina propria and circular muscle layer of uterus; B. Positive staining for FSHR was observed in the mucous plica and circular muscle layer of the oviduct; C. Positive staining for FSHR was observed in the stratum granulosum of the uterus. Second-line: A, A-1. Positive staining for GHR was observed in epithelial cell and muscular layer of uterus; B. Positive staining for GHR was observed in the mucous plica and circular muscle layer of the oviduct; C. Positive staining for GHR was observed in the stratum granulosum and theca externa of follicle of the uterus. Third-line: A. Positive staining for LHR was observed in epithelial cell and muscular layer of uterus; B. Positive staining for LHR was observed in the mucous plica and circular muscle layer of the oviduct; C, C-1. Positive staining for LHR was observed in the stratum granulosum and theca externa of follicle of the uterus; EC — epithelial cell; CML — circular muscle layer; LP — lamina propria; MP — mucous plica; SG — stratum granulosum; TFI — theca interna of follicle; TFE — theca externa of follicle; $\mathrm{CO}$ - cumulus oophorus; FA — follicular antrum. 
Table 3. Quantitative real-time polymerase chain reaction (qRT-PCR) results of FSHR/GHR/LHR gene in reproductive system of yak

\begin{tabular}{lccc}
\hline Tissues and organs & $\Delta \mathbf{C T}$ & $\Delta \Delta \mathbf{C T}\left(\Delta \mathbf{C T}_{\text {sample }}-\Delta \mathbf{C T}_{\text {ovary/oviduct }}\right)$ & $\mathbf{2}^{-\Delta \Delta \mathbf{C T}}$ \\
\hline $\boldsymbol{F S H R}$ & & & 8.2059 \\
Uterus & $-8.9367 \pm 0.2435$ & $-3.0367 \pm 0.2435$ & 4.8502 \\
Oviduct & $2.3080 \pm 0.1567$ & $-2.2781 \pm 0.1567$ & 1 \\
Ovary & $-5.9000 \pm 0.1107$ & $0 \pm 0.1107$ & 6.4218 \\
GHR & & & 7.0267 \\
Uterus & $-8.5830 \pm 0.1127$ & $-2.6830 \pm 0.1127$ & 3.8937 \\
Oviduct & $-8.7129 \pm 0.2335$ & $-2.8129 \pm 0.2335$ & 2.1835 \\
Ovary & $-7.8611 \pm 0.2472$ & $-1.9611 \pm 0.2472$ & 2.0897 \\
LHR & & & 1.7639 \\
Uterus & $-7.0267 \pm 0.1346$ & $-1.1267 \pm 0.1346$ & $-1.0633 \pm 0.1657$ \\
Oviduct & $-6.9633 \pm 0.1657$ & $-0.8188 \pm 0.1094$ & \\
Ovary & $-6.7188 \pm 0.1094$ & & \\
\hline
\end{tabular}

FSHR — follicle stimulating hormone receptor; GHR — growth hormone receptor; LHR — luteinising hormone receptor

mainly expressed in the pituitary gland and uterus. FSHR and LHR were located in the pineal gland, hypothalamus, pituitary, and gonad oestrus of female yaks [8]. Recent study elucidated the influence of two different $\mathrm{FSH}$ additives on in vitro maturation rate and gene expression of cumulus-oocytes complexes in domestic cat. However, variations were discovered in correlations of expression levels, for instance for FSHR and LHCG, indicating differences in the finetuning of in vitro maturation processes under varying FSH supplementations [26]. In the present study, the gene expression levels of FSHR and LHR in tissues were similar, but there were differences in expression levels of the three proteins. The highest expression levels of FSHR and LHR were in the kidney, cerebellum and lung of female yaks. Therefore, the results suggest that the high expression levels of FSHR and LHR contribute to the metabolism of organisms.

In the present study, the expression level of GHR are the highest in the kidney, cerebellum, lung, spleen, and liver. It is reported that a novel phenotype for Laron dwarfism in Bos indicus cattle was associated with underexpression of GHR 1A mRNA, but not other GHR mRNA variants in the liver [14]. The study of Zeng et al. [27] showed that GRH 1 and 2 were correlated with each other in adult tissues of blunt snout bream and megalobrama amblycephala. During development, GHR 1 had a similar expression pattern to GHR 2 during the examination. It can be proved that GHR plays an active role in tissue physiological characteristics of female yaks.
Our study showed for the first time that FSHR/ GHR/LHR are localised in different tissues of female yaks. The $L 2 \beta$ loop of marmoset and human FSH has distinct receptor interaction ability and immunoreactivity indicating possibility of subtle conformational and biochemical differences between the two regions which may affect the FSH-FSHR interaction in these two primates [12]. It has been proved that the main location of FSHR/GHR/LHR is in kidney tubules, Purkinje cells, cerebellar medulla, and alveolar cells of lung, whereas the almost no positive reaction was observed in liver and spleen. Previous reports showed that the GHR gene not only provided confirmatory evidences for previous findings, but also explored a suite of novel SNP associated with milk production traits, and thus formed a solid foundation for eventually unravelling the causal mutations for milk production traits in dairy cattle [9]. Considering our findings with those reported in different tissues of the female yaks during luteal phase, we speculate that the expression levels of FSHR/GHR/GHR are regulated by protective tissues.

In this study, we determined that the expression levels of FSHR/GHR/LHR were significantly different in the female reproductive organs of the uterus, oviduct and ovary of female yaks during luteal phase $(p<0.01)$. Effect of follicle size on mRNA expression was reported in Bos indicus cumulus cells and oocytes. In cumulus cells, the expression levels of FSHR, EGFR and GHR mRNA increased with follicular size [3]. FSH can accelerate the maturation rate of sheep oocytes and reduce the rate of apoptosis, but also increase the 
expression levels of FSHR, LHR and GnRHR mRNAs, strengthen expressions of FSHR and GnRHR proteins [21]. Variable expression are observed in almost all of the genes in the present study at high and low fecundity, and the FSHR record was the most significant for the high and low fecundity of the ovarian follicles of Indian sheep ewes [5]. However, animal studies previously showed that LHR and FSHR were important regulators in prepubertal Bos indicus ovarian tissues and postpubertal heifers in dioestrus [24]. In the present study, we found that the FSHR/GHR/ /LHR expression levels were higher in the uterus and oviduct, and weaker expression in FSHR/GHR in the ovaries. These finding suggest that FSHR/GHR levels increase within these tissues of the body due to the luteal phase of the female yaks. However, the expression of LHR is not evident in the female reproductive organs and the luteal phase of female yaks

This study demonstrated the localisation of FSHR/ GHR/GHR in the cell membrane and cytoplasm organs, not the nuclei. However, animal studies showed that the FSHR is present in horse ovaries constantly from birth, throughout life, and expressed in the oestrous cycle. FSHR protein was found in granulosa cells, cumulus cells and inconsistently in oocytes, independent of animal age or folliculogenesis stage [19]. However, this test demonstrated that the main locations of FSHR/GHR/LHR were in muscular layer and intima of uterus, mucous membrane of oviduct, granulosa cells of ovary. There was no location of GHR in the lamina propria of the uterus. These finding suggest that FSHR/GHR/LHR positions are tagged in these tissues of the body due to the reproductive organs of the female yaks during luteal phase. Immunohistochemical staining revealed the presence of BMPR1B but not the primordial follicles of FSH receptor (FSHR). Consistent with previous studies, the development of BMP signalling system can be an effective approach to maintain the primordial follicle reserve while promoting follicular growth, ovulation and consequently overall female fertility [1]. Therefore, the regulative function of the FSHR/GHR/ /LHR might relate to the development of BMP signalling system. It is further implied that the FSHR/GHR/ /LHR play important roles in the development of the luteal and process reproduction with regulation.

\section{CONCLUSIONS}

The following conclusions can be drawn from this study.
FSHR/GHR/LHR have distinct differential expressions in different tissues, and are especially highly expressed in the kidney, cerebellum. FSHR/GHR/LHR are located in kidney tubules, Purkinje cells, cerebellar medulla, alveolar cells and hepatocytes.

The expression levels of FSHR and GHR are consistently higher than that of LHR in the reproductive organs of female yaks during luteal phase. Meanwhile, FSHR/GHR/LHR are located in the cardiac muscle cells, cerebellar medulla, theca cells lining.

The expression level of FSHR is slightly higher than that of GHR and LHR in all examined tissues.

\section{REFERENCES}

1. Al-Samerria S, Al-Ali I, McFarlane JR, et al. The impact of passive immunisation against BMPRIB and BMP4 on follicle development and ovulation in mice. Reproduction. 2015; 149(5): 403-411, doi: 10.1530/REP-14-0451, indexed in Pubmed: 25667430.

2. Ayoub MA, Yvinec $R$, Jégot $G$, et al. Profiling of FSHR negative allosteric modulators on $L H / C G R$ reveals biased antagonism with implications in steroidogenesis. Mol Cell Endocrinol. 2016; 436: 10-22, doi: 10.1016/j. mce.2016.07.013, indexed in Pubmed: 27424143.

3. Caixeta ES, Ripamonte P, Franco MM, et al. Effect of follicle size on mRNA expression in cumulus cells and oocytes of Bos indicus: an approach to identify marker genes for developmental competence. Reprod Fertil Dev. 2009; 21(5): 655-664, doi: 10.1071/RD08201, indexed in Pubmed: 19486602.

4. Du X, Li Q, Pan Z, et al. Androgen receptor and miRNA-126* axis controls follicle-stimulating hormone receptor expression in porcine ovarian granulosa cells. Reproduction. 2016; 152(2): 161-169, doi: 10.1530/REP15-0517, indexed in Pubmed: 27222597.

5. Goyal S, Aggarwal J, Dubey PK, et al. Expression Analysis of Genes Associated with Prolificacy in FecB Carrier and Noncarrier Indian Sheep. Anim Biotechnol. 2017; 28(3): 220-227, doi: 10.1080/10495398.2016.1262869, indexed in Pubmed: 28075701.

6. Gu W, Yang L, Wang S, et al. Generation and application of a novel InsP(3)R(1) mono-antibody from mouse. J Immunoassay Immunochem. 2015; 36(5): 487-495, doi: 10.1080/ 15321819.2014.996817, indexed in Pubmed: 25522905.

7. He Y, Yu S, Hu J, et al. Changes in the Anatomic and Microscopic Structure and the Expression of HIF-1 $\alpha$ and VEGF of the Yak Heart with Aging and Hypoxia. PLoS One. 2016; 11(2): e0149947, doi: 10.1371/journal.pone.0149947, indexed in Pubmed: 26914488.

8. Huo SD, Chen SE, Long RJ, et al. Protein and mRNA expression of follicle-stimulating hormone receptor and luteinizing hormone receptor during the oestrus in the yak (Bos grunniens). Reprod Domest Anim. 2017; 52(3): 477-482, doi: 10.1111/rda.12936, indexed in Pubmed: 28181328.

9. Jiang Li, Liu J, Sun D, et al. Genome wide association studies for milk production traits in Chinese Holstein population. PLoS One. 2010; 5(10): e13661, doi: 10.1371/ journal.pone.0013661, indexed in Pubmed: 21048968. 
10. Juengel JL, French MC, Quirke LD, et al. Differential expression of CART in ewes with differing ovulation rates. Reproduction. 2017; 153(4): 471-479, doi: 10.1530/REP16-0657, indexed in Pubmed: 28115581.

11. Kareem KY, Loh TC, Foo HL, et al. Effects of dietary postbiotic and inulin on growth performance, IGF1 and GHR mRNA expression, faecal microbiota and volatile fatty acids in broilers. BMC Vet Res. 2016; 12(1): 163, doi: 10.1186/ s12917-016-0790-9, indexed in Pubmed: 27496016.

12. Kutteyil SS, Kulkarni BJ, Mojidra R, et al. Comparison of marmoset and human FSH using synthetic peptides of the -subunit L2 loop region and anti-peptide antibodies. J Pept Sci. 2016; 22(6): 397-405, doi: 10.1002/psc.2882, indexed in Pubmed: 27282136.

13. Lei ZM, Toth P, Rao CV, et al. Novel coexpression of human chorionic gonadotropin (hCG)/human luteinizing hormone receptors and their ligand hCG in human fallopian tubes. J Clin Endocrinol Metab. 1993; 77(3): 863-872, doi: 10.1210/jc.77.3.863.

14. Liu J, Boyd CK, Kobayashi Y, et al. A novel phenotype for Laron dwarfism in miniature Bos indicus cattle suggests that the expression of growth hormone receptor $1 \mathrm{~A}$ in liver is required for normal growth*. Domestic Animal Endocrinology. 1999; 17(4): 421-437, doi: 10.1016/s07397240(99)00051-x.

15. Marson EP, Ferraz JBS, Meirelles FV, et al. Effects of polymorphisms of LHR and FSHR genes on sexual precocity in a Bos taurus $x$ Bos indicus beef composite population. Genet Mol Res. 2008; 7(1): 243-251, indexed in Pubmed: 18393228.

16. Ohkubo T, Yano H, Takahashi S, et al. Bos indicus type of growth hormone receptor gene is retained in Japanese Black cattle. J Anim Breed Genet. 2006; 123(6): 410-413, doi: 10.1111/j.1439-0388.2006.00617.x, indexed in Pubmed: 17177699.

17. Parrott J, Doraiswamy V, Kim G, et al. Expression and actions of both the follicle stimulating hormone receptor and the luteinizing hormone receptor in normal ovarian surface epithelium and ovarian cancer. Mol Cell Endocrinol. 2001; 172(1-2): 213-222, doi: 10.1016/s03037207(00)00340-3.

18. Qi HL, Li CS, Qian CW, et al. The long noncoding RNA, EGFR-AS1, a target of GHR, increases the expression of EGFR in hepatocellular carcinoma. Tumour Biol. 2016; 37(1): 1079-1089, doi: 10.1007/s13277-015-3887-z, indexed in Pubmed: 26271667.
19. Scarlet D, Walter I, Hlavaty J, et al. Expression and immunolocalisation of follicle-stimulating hormone receptors in gonads of newborn and adult female horses. Reprod Fertil Dev. 2016 [Epub ahead of print]; 28(9): 1340-1348, doi: 10.1071/RD14392, indexed in Pubmed: 25693905.

20. Schneider A, Pfeifer L, Hax L, et al. Insulin-like growth factor and growth hormone receptor in postpartum lactating beef cows. Pesquisa Agropecuária Brasileira. 2010; 45(8): 925-931, doi: 10.1590/s0100-204×2010000800019.

21. Suocheng W, Zhuandi G, Li S, et al. Maturation rates of oocytes and levels of FSHR, LHR and GnRHR of COCs response to FSH concentrations in IVM media for sheep. J Applied Biomed. 2017; 15(3): 180-186, doi: 10.1016/j. jab.2017.01.001.

22. Wang W, Liu S, Li F, et al. Polymorphisms of the Ovine BMPR-IB, BMP-15 and FSHR and Their Associations with Litter Size in Two Chinese Indigenous Sheep Breeds. Int J Mol Sci. 2015; 16(5): 11385-11397, doi: 10.3390/ ijms160511385, indexed in Pubmed: 25993301.

23. Wei S, Gong Z, An L, et al. Cloprostenol and pregnant mare serum gonadotropin promote estrus synchronization, uterine development, and follicle-stimulating hormone receptor expression in mice. Genet Mol Res. 2015; 14(2): 7184-7195, doi: 10.4238/2015.June.29.12, indexed in Pubmed: 26125929.

24. Weller M, Fortes M, Porto-Neto L, et al. Candidate gene expression in bos indicus ovarian tissues: prepubertal and postpubertal heifers in diestrus. Frontiers Vet Scien. 2016; 3, doi: 10.3389/fvets.2016.00094.

25. Wiener G, Jianlin H, Ruijun L. The Yak.: FAO Regional Office for Asia and the Pacific Food and Agriculture Organization of the United Nations. Bangkok. Thailand; 2003.

26. Zahmel J, Mundt H, Jewgenow K, et al. Analysis of gene expression in granulosa cells post-maturation to evaluate oocyte culture systems in the domestic cat. Reprod Domest Anim. 2017; 52 Suppl 2: 65-70, doi: 10.1111/rda.12927, indexed in Pubmed: 28120353.

27. Zeng $C$, Liu XI, Wang Wm, et al. Characterization of GHRs, IGFs and MSTNs, and analysis of their expression relationships in blunt snout bream, Megalobrama amblycephala. Gene. 2014; 535(2): 239-249, doi: 10.1016/j. gene.2013.11.027.

28. Zheng M, Shi H, Segaloff $D$, et al. Expression and localization of luteinizing hormone receptor in the female mouse reproductive tract. Biol Reprod. 2001; 64(1): 179-187, doi: 10.1093/biolreprod/64.1.179. 